\title{
A GAME THEORETICAL APPROACH TO THE ALGEBRAIC COUNTERPART OF THE WAGNER HIERARCHY: PART I
}

\author{
JÉRÉmie CABEssa ${ }^{1}$ AND JACQUES DuparC ${ }^{1}$
}

\begin{abstract}
The algebraic study of formal languages shows that $\omega$ rational sets correspond precisely to the $\omega$-languages recognizable by finite $\omega$-semigroups. Within this framework, we provide a construction of the algebraic counterpart of the Wagner hierarchy. We adopt a hierarchical game approach, by translating the Wadge theory from the $\omega$-rational language to the $\omega$-semigroup context. More precisely, we first show that the Wagner degree is indeed a syntactic invariant. We then define a reduction relation on finite pointed $\omega$-semigroups by means of a Wadge-like infinite two-player game. The collection of these algebraic structures ordered by this reduction is then proven to be isomorphic to the Wagner hierarchy, namely a well-founded and decidable partial ordering of width 2 and height $\omega^{\omega}$.
\end{abstract}

Mathematics Subject Classification. O3D55, 20M35, 68Q70, 91A65.

\section{INTRODUCTION}

This paper is the first part of a series of two. Its content lies at the crossroads of two mathematical fields, namely the algebraic theory of $\omega$-automata, and hierarchical games, in descriptive set theory.

The basic interest of the algebraic approach to automata theory consists in the equivalence between Büchi automata and some structures extending the notion of a semigroup, called $\omega$-semigroups [13]. These mathematical objects indeed satisfy several relevant properties. Firstly, given a finite Büchi automaton, one

\footnotetext{
Keywords and phrases. $\omega$-automata, $\omega$-rational languages, $\omega$-semigroups, infinite games, hierarchical games, Wadge game, Wadge hierarchy, Wagner hierarchy.

1 University of Lausanne, Faculty of Business and Economics, HEC - ISI, 1015 Lausanne, Switzerland; Jeremie.Cabessa@unil.ch
} 
can effectively compute a finite $\omega$-semigroup recognizing the same $\omega$-language, and vice versa. Secondly, among all finite $\omega$-semigroups recognizing a given $\omega$ language, there exists a minimal one - called the syntactic $\omega$-semigroup -, whereas there is no convincing notion of Büchi (or Muller) minimal automaton. Thirdly, finite $\omega$-semigroup appear to be a powerful tool towards the classification of $\omega$ rational languages: for instance, an $\omega$-language is first-order definable if and only if it is recognized by an aperiodic $\omega$-semigroup [8,11,19], a generalization to infinite words of Schützenberger, and McNaughton and Papert's famous results $[10,17]$. Even some topological properties (being open, closed, clopen, $\boldsymbol{\Sigma}_{\mathbf{2}}^{\mathbf{0}}, \boldsymbol{\Pi}_{\mathbf{2}}^{\mathbf{0}}, \boldsymbol{\Delta}_{\mathbf{2}}^{\mathbf{0}}$ ) can be characterized by algebraic properties on $\omega$-semigroups (see [15] or [13], Chap. 3).

Hierarchical games, for their part, aim to classify subsets of topological spaces, in particular by means of the following Wadge reduction: given two topological spaces $E$ and $F$, and two subsets $X \subseteq E$ and $Y \subseteq F$, the set $X$ is said to be Wadge reducible to $Y$ iff there exists a continuous function from $E$ into $F$ such that $X=f^{-1}(Y)$, or equivalently, iff there exists a winning strategy for Player II in the Wadge game $\mathbb{W}(X, Y)[20,21]$. The resulting Wadge hierarchy - the most refined hierarchy in descriptive set theory - appeared to be specially interesting to computer scientists, for it illuminates the study of classifying $\omega$-rational languages. In this context, two main questions arise when $X$ Wadge reduces to $Y$ :

- Effectivity: if $X$ and $Y$ are given effectively, is it then possible to provide an effective computation of a continuous function $f$ such that $X=f^{-1}(Y)$ ?

- Automaticity: if $X$ and $Y$ are recognized by finite $\omega$-automata, is there also an automatic ${ }^{1}$ continuous function $f$ such that $X=f^{-1}(Y)$ ?

An extended literature exists on both questions. In particular, Klaus Wagner answered positively to the second problem [22], and the restriction of the Wadge hierarchy to $\omega$-rational sets is in fact entirely known: it coincides precisely with the original Wagner hierarchy - the most refined classification of $\omega$-rational sets -, namely a well-founded and decidable partial ordering of width 2 and height $\omega^{\omega}$. The Wagner degree of any $\omega$-rational set is furthermore efficiently computable [24].

Wagner's original proofs rely on a graph-theoretic analysis of Muller automata, away from the algebraic framework. Carton and Perrin [3-5] investigated the algebraic reformulation of the Wagner hierarchy, a work carried on by Duparc and Riss [7]. However, this new approach is not yet entirely satisfactory, for it fails to provide an algorithm computing the Wagner degree of any $\omega$-rational set directly on its syntactic $\omega$-semigroup.

Our papers fill this gap. We first show by a direct argument that the Wagner degree is indeed a syntactic invariant. We then define a reduction on subsets of finite $\omega$-semigroups by means of an infinite game, without any direct reference to the Wagner hierarchy. We show that the resulting algebraic hierarchy is isomorphic to the Wagner hierarchy, and in this sense corresponds to the algebraic counterpart of the Wagner hierarchy, In particular, this classification is a refinement of the hierarchies of chains and superchains introduced in $[3,5]$. Moreover, we prove that

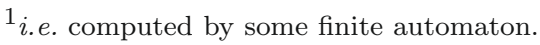


the Wagner degree of any given subset of a finite $\omega$-semigroup can be effectively computed. The detailed description of this decidability procedure is given in the second paper.

\section{Preliminaries}

\subsection{LANGUAGES}

Given a finite set $A$, called the alphabet, then $A^{*}, A^{+}, A^{\omega}$, and $A^{\infty}$ denote respectively the sets of finite words, nonempty finite words, infinite words, and finite or infinite words, all of them over the alphabet $A$. The empty word is denoted by $\varepsilon$. Given a finite word $u$ and a finite or infinite word $v$, then $u v$ denotes the concatenation of $u$ and $v$. Given $X \subseteq A^{*}$ and $Y \subseteq A^{\infty}$, the concatenation of $X$ and $Y$ is defined by $X Y=\{x y \mid x \in X$ and $y \in Y\}$, the finite iteration of $X$ by $X^{*}=\left\{x_{1} \ldots x_{n} \mid n \geq 0\right.$ and $\left.x_{1}, \ldots, x_{n} \in X\right\}$, and the infinite iteration of $X$ by $X^{\omega}=\left\{x_{0} x_{1} x_{2} \ldots \mid x_{i} \in X\right.$, for all $\left.i \in \mathbb{N}\right\}$.

We refer to [13], p. 15 , for the definition of $\omega$-rational languages. The $\omega$-rational languages are exactly the ones recognized by finite Büchi, or equivalently, by finite Muller automata [13].

\subsection{Semigroups}

A semigroup $(S, \cdot)$ is a set $S$ equipped with an associative binary operation on $S$. When equipped with an identity element, a semigroup becomes a monoid. If $S$ is a semigroup, then $S^{1}$ denotes $S$ if $S$ is a monoid, and $S \cup\{1\}$ otherwise, with the operation of $S$ completed by the relations $1 \cdot x=x \cdot 1=x$, for every $x \in S^{1}$. A semigroup morphism is a map $\varphi$ from a semigroup $S$ into a semigroup $T$ such that $\varphi\left(s_{1} \cdot s_{2}\right)=\varphi\left(s_{1}\right) \cdot \varphi\left(s_{2}\right)$, for every $s_{1}, s_{2} \in S$. A semigroup congruence on $S$ is an equivalence relation $\sim$ such that for every $s, t \in S$ and every $x, y \in S^{1}$, the condition $s \sim t$ implies $x s y \sim x t y$. The quotient set $S / \sim$ is naturally equipped with a structure of semigroup, and the function which maps every element onto its $\sim$-class is a semigroup morphism from $S$ onto $S / \sim$.

\section{3. $\omega$-SEMIGROUPS}

The notion of an $\omega$-semigroup was first introduced by Pin as a generalization of semigroups $[12,14]$. In the case of finite structures, these objects represent a convincing algebraic counterpart to automata reading infinite words: given any finite Büchi automaton, one can build a finite $\omega$-semigroup recognizing (in an algebraic sense) the same language, and conversely, given any finite $\omega$-semigroup recognizing a certain language, one can build a finite Büchi automaton recognizing the same language. 
Definition 1.1 (see [13], p. 92). An $\omega$-semigroup is an algebra consisting of two components, $S=\left(S_{+}, S_{\omega}\right)$, and equipped with the following operations:

- a binary operation on $S_{+}$, denoted multiplicatively, such that $S_{+}$equipped with this operation is a semigroup;

- a mapping $S_{+} \times S_{\omega} \longrightarrow S_{\omega}$, called mixed product, which associates with each pair $(s, t) \in S_{+} \times S_{\omega}$ an element of $S_{\omega}$, denoted by $s t$, and such that for every $s, t \in S_{+}$and for every $u \in S_{\omega}$, then $s(t u)=(s t) u$;

- a surjective mapping $\pi_{S}: S_{+}^{\omega} \longrightarrow S_{\omega}$, called infinite product, which is compatible with the binary operation on $S_{+}$and the mixed product in the following sense: for every strictly increasing sequence of integers $\left(k_{n}\right)_{n>0}$, for every sequence $\left(s_{n}\right)_{n \geq 0} \in S_{+}^{\omega}$, and for every $s \in S_{+}$, then

$$
\begin{gathered}
\pi_{S}\left(s_{0} s_{1} \ldots s_{k_{1}-1}, s_{k_{1}} \ldots s_{k_{2}-1}, \ldots\right)=\pi_{S}\left(s_{0}, s_{1}, s_{2}, \ldots\right) \\
s \pi_{S}\left(s_{0}, s_{1}, s_{2}, \ldots\right)=\pi_{S}\left(s, s_{0}, s_{1}, s_{2}, \ldots\right) .
\end{gathered}
$$

Intuitively, an $\omega$-semigroup is a semigroup equipped with a suitable infinite product. The conditions on the infinite product ensure that one can replace the notation $\pi_{S}\left(s_{0}, s_{1}, s_{2}, \ldots\right)$ by the notation $s_{0} s_{1} s_{2} \ldots$ without ambiguity. Since an $\omega$-semigroup is a pair $\left(S_{+}, S_{\omega}\right)$, it is convenient to call + -subsets and $\omega$-subsets the subsets of $S_{+}$and $S_{\omega}$, respectively.

Given two $\omega$-semigroups $S=\left(S_{+}, S_{\omega}\right)$ and $T=\left(T_{+}, T_{\omega}\right)$, a morphism of $\omega$ semigroups from $S$ into $T$ is a pair $\varphi=\left(\varphi_{+}, \varphi_{\omega}\right)$, where $\varphi_{+}: S_{+} \longrightarrow T_{+}$is a morphism of semigroups, and $\varphi_{\omega}: S_{\omega} \longrightarrow T_{\omega}$ is a mapping canonically induced by $\varphi_{+}$in order to preserve the infinite product, that is, for every sequence $\left(s_{n}\right)_{n \geq 0}$ of elements of $S_{+}$, then

$$
\varphi_{\omega}\left(\pi_{S}\left(s_{0}, s_{1}, s_{2}, \ldots\right)\right)=\pi_{T}\left(\varphi_{+}\left(s_{0}\right), \varphi_{+}\left(s_{1}\right), \varphi_{+}\left(s_{2}\right), \ldots\right) .
$$

An $\omega$-semigroup $S$ is an $\omega$-subsemigroup of $T$ if there exists an injective morphism of $\omega$-semigroups from $S$ into $T$. An $\omega$-semigroup $S$ is a quotient of $T$ if there exists a surjective morphism of $\omega$-semigroups from $T$ onto $S$. An $\omega$-semigroup $S$ divides $T$ if $S$ is quotient of an $\omega$-subsemigroup of $T$.

The notion of pointed $\omega$-semigroup can adapted from the notion of pointed semigroup introduced by Sakarovitch [16]. In this paper, a pointed $\omega$-semigroup denotes a pair $(S, X)$, where $S$ is an $\omega$-semigroup and $X$ is an $\omega$-subset of $S$. The pair $\left(S, X^{c}\right)$ will then stand for the pointed $\omega$-semigroup $\left(S, S_{\omega} \backslash X\right)$. A mapping $\varphi:(S, X) \longrightarrow(T, Y)$ is a morphism of pointed $\omega$-semigroups if $\varphi: S \longrightarrow T$ is a morphism of $\omega$-semigroups such that $\varphi^{-1}(Y)=X$. The notions of $\omega$ subsemigroups, quotient, and division can then be easily adapted in the context of pointed $\omega$-semigroups.

A congruence of an $\omega$-semigroup $S=\left(S_{+}, S_{\omega}\right)$ [13] is a pair $\left(\sim_{+}, \sim_{\omega}\right)$, where $\sim_{+}$is a semigroup congruence on $S_{+}, \sim_{\omega}$ is an equivalence relation on $S_{\omega}$, and these relations are stable for the infinite and the mixed products: if $\left(s_{0}, s_{1}, \ldots\right)$ and $\left(t_{0}, t_{1}, \ldots\right)$ are sequences of elements of $S_{+}$such that $s_{i} \sim_{+} t_{i}$, for each $i \geq 0$, then $s_{0} s_{1} s_{2} \ldots \sim_{\omega} t_{0} t_{1} t_{2} \ldots$, and if $s, s^{\prime} \in S_{+}$and $x, x^{\prime} \in S_{\omega}$ such that $s \sim_{+} s^{\prime}$ 
and $x \sim_{\omega} x^{\prime}$, then $s x \sim_{\omega} s^{\prime} x^{\prime}$. The quotient set $S / \sim=\left(S / \sim_{+}, S / \sim_{\omega}\right)$ is naturally equipped with a structure of $\omega$-semigroup. If $\left(\sim_{i}\right)_{i \in I}$ is a family of congruences on an $\omega$-semigroup, then the congruence $\sim$, defined by $s \sim t$ if and only if $s \sim_{i} t$ for all $i \in I$, is called the lower bound of the family $\left(\sim_{i}\right)_{i \in I}$. The upper bound of the family $\left(\sim_{i}\right)_{i \in I}$ is then the lower bound of the congruences that are coarser than all the $\sim_{i}$.

Example 1.2. The trivial $\omega$-semigroup, denoted by $1=(\{1\},\{a\})$, is obtained by equipping the trivial semigroup $\{1\}$ with the infinite product $\pi$ defined by $\pi(1,1,1, \ldots)=a$.

Example 1.3. Let $A$ be an alphabet. The $\omega$-semigroup $A^{\infty}=\left(A^{+}, A^{\omega}\right)$ equipped with the usual concatenation is the free $\omega$-semigroup over the alphabet $A$ [3].

Example 1.4. Let $S=\left(S_{+}, S_{\omega}\right)$ be a finite $\omega$-semigroup. The morphism of $\omega$ semigroups $\varphi: S_{+}^{\infty} \longrightarrow S$ naturally induced by the identity over $S_{+}$is called the canonical morphism associated with $S$.

In this paper, we strictly focus on finite $\omega$-semigroups, those whose first component is finite. It is proven in [13] that the infinite product $\pi_{S}$ of a finite $\omega$-semigroup $S$ is completely determined be the mixed products of the form $x \pi_{S}(s, s, s, \ldots)$ (denoted $\left.x s^{\omega}\right)$. We use this property in the next examples, also taken from [13].

Example 1.5. The pair $S=\left(\{0,1\},\left\{0^{\omega}, 1^{\omega}\right\}\right)$ is an $\omega$-semigroup for the operations defined as follows:

$$
\begin{array}{llll}
0 \cdot 0=0 & 0 \cdot 1=0 & 1 \cdot 0=0 & 1 \cdot 1=1 \\
00^{\omega}=0^{\omega} & 10^{\omega}=0^{\omega} & 01^{\omega}=1^{\omega} & 11^{\omega}=1^{\omega} .
\end{array}
$$

Example 1.6. The pair $T=\left(\{a, b, c, c a\},\left\{a^{\omega},(c a)^{\omega}, 0\right\}\right)$ is an $\omega$-semigroup for the operations defined as follows:

$$
\begin{aligned}
& a^{2}=a \quad a b=a \quad a c=a \quad b a=a \\
& b^{2}=b \quad b c=c \quad c b=c \quad c^{2}=c \\
& b^{\omega}=a^{\omega} \quad c^{\omega}=0 \quad a a^{\omega}=a^{\omega} \quad a(c a)^{\omega}=a^{\omega} \\
& b a^{\omega}=a^{\omega} \quad b(c a)^{\omega}=(c a)^{\omega} \quad c a^{\omega}=(c a)^{\omega} \quad c(c a)^{\omega}=(c a)^{\omega} .
\end{aligned}
$$

Wilke was the first to give the appropriate algebraic counterpart to finite automata reading infinite words [23]. In addition, he established that the $\omega$-languages recognized by finite $\omega$-semigroups are exactly the ones recognized by Büchi automata, a proof that can be found in [23] or [13].

Definition 1.7. Let $S$ and $T$ be two $\omega$-semigroups. One says that a surjective morphism of $\omega$-semigroups $\varphi: S \longrightarrow T$ recognizes a subset $X$ of $S$ if there exists a subset $Y$ of $T$ such that $\varphi^{-1}(Y)=X$. By extension, one also says in this case that the $\omega$-semigroup $T$ recognizes $X$. In addition, a congruence $\sim$ on $S$ recognizes the subset $X$ of $S$ if the natural morphism $\pi: S \longrightarrow S / \sim$ recognizes $X$. 
Proposition 1.8 (Wilke). An $\omega$-language is recognized by a finite $\omega$-semigroup if and only if it is $\omega$-rational.

Example 1.9. Let $A=\{a, b\}$, let $S$ be the $\omega$-semigroup given in Example 1.5, and let $\varphi: A^{\infty} \longrightarrow S$ be the morphism defined by $\varphi(a)=0$ and $\varphi(b)=1$. Then $\varphi^{-1}\left(0^{\omega}\right)=\left(A^{*} a\right)^{\omega}$ and $\varphi^{-1}\left(1^{\omega}\right)=A^{*} b^{\omega}$.

\subsection{TOPOLOGY}

For any set $A$, the set $A^{\omega}$ can be equipped with the product topology of the discrete topology on $A$. The open sets of $A^{\omega}$ are of the form $W A^{\omega}$, for some $W \subseteq A^{*}$. Given a topological space $E$, the class of Borel subsets of $E$ is the smallest class containing the open sets, and closed under countable union and complementation. Flip sets are samples of non-Borel sets: a subset $F$ of $\{0,1\}^{\omega}$ is a flip set [1] if changing one bit of any infinite word shifts it from $F$ to its complement, or vice versa; more precisely, if the following formula holds

$$
\forall x, y \in\{0,1\}^{\omega}(\exists ! k \geq 0(x(k) \neq y(k))) \Rightarrow(x \in F \Leftrightarrow y \notin F) .
$$

No flip set is Borel, since Borel sets satisfy the Baire property, whereas flip sets do not [1]. Finally, for any set $X$ and any index $i \in\{0,1\}$, one sets

$$
X^{c(i)}= \begin{cases}X & \text { if } i=0 \\ X^{c} & \text { if } i=1\end{cases}
$$

In addition, a pointed $\omega$-semigroup $(S, X)$ will be called Borel if the preimage $\pi_{S}^{-1}(X)$ is a Borel subset of $S_{+}^{\omega}$ (where $S_{+}^{\omega}$ is equipped with the product topology of the discrete topology on $\left.S_{+}\right)$. Therefore, every finite pointed $\omega$-semigroup is Borel, since, by Proposition 1.8, its preimage by the infinite product is $\omega$-rational, hence Borel.

\subsection{The Wadge hierarchy}

Let $A$ and $B$ be two alphabets, and let $X \subseteq A^{\omega}$ and $Y \subseteq B^{\omega}$. The Wadge game $\mathbb{W}((A, X),(B, Y))[20]$ is a two-player infinite game with perfect information, where Player I is in charge of the subset $X$ and Player II is in charge of the subset $Y$. Players I and II alternately play letters from the alphabets $A$ and $B$, respectively. Player I begins. Player II is allowed to skip her turn - formally denoted by the symbol "-" - provided she plays infinitely many letters, whereas Player I is not allowed to do so. After $\omega$ turns each, players I and II respectively produced two infinite words $\alpha \in A^{\omega}$ and $\beta \in B^{\omega}$. Player II wins $\mathbb{W}((A, X),(B, Y))$ if and only if $(\alpha \in X \Leftrightarrow \beta \in Y)$. From this point onward, the Wadge game $\mathbb{W}((A, X),(B, Y))$ will be denoted $\mathbb{W}(X, Y)$ and the alphabets involved will always be clear from the 
context. A play of this game is illustrated below.

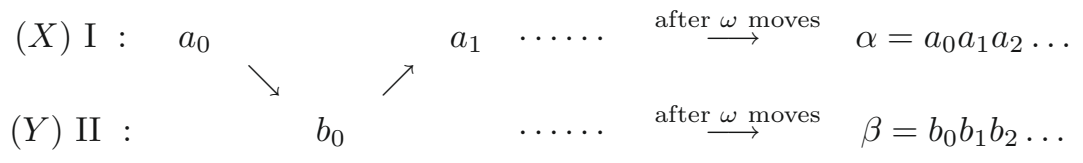

Along the play, the finite sequence of all previous moves of a given player is called the current position of this player. A strategy for Player I is a mapping from $(B \cup\{-\})^{*}$ into $A$. A strategy for Player II is a mapping from $A^{+}$into $B \cup\{-\}$. A strategy is winning if the player following it must necessarily win, no matter what his opponent plays.

The Wadge reduction is defined via the Wadge game as follows: a set $X$ is said to be Wadge reducible to $Y$, denoted by $X \leq_{W} Y$, if and only if Player II has a winning strategy in $\mathbb{W}(X, Y)$. One then sets $X \equiv_{W} Y$ if and only if both $X \leq_{W} Y$ and $Y \leq_{W} X$, and also $X<_{W} Y$ if and only if $X \leq_{W} Y$ and $X \not_{W} Y$. The relation $\leq_{W}$ is reflexive and transitive, and $\equiv_{W}$ is an equivalence relation. A set $X$ is called self-dual if $X \equiv_{W} X^{c}$, and non-self-dual if $X \not_{W} X^{c}$. One can show [21] that the Wadge reduction coincides with the continuous reduction, that is $X \leq_{W} Y$ if and only if $f^{-1}(Y)=X$, for some continuous function $f: A^{\omega} \longrightarrow B^{\omega}$.

The Wadge hierarchy consists of the collection of all $\omega$-languages ordered by the Wadge reduction, and the Borel Wadge hierarchy is the restriction of the Wadge hierarchy to Borel $\omega$-languages. Martin's Borel determinacy [9] easily implies Borel Wadge determinacy, that is, whenever $X$ and $Y$ are Borel sets, then one of the two players has a winning strategy in $\mathbb{W}(X, Y)$. This key property induces strong consequences on the Borel Wadge hierarchy: the $\leq_{W}$-antichains have length at most 2; the only incomparable $\omega$-languages are (up to Wadge equivalence) of the form $X$ and $X^{c}$, for $X$ non-self-dual; furthermore, the Wadge reduction is well-founded on Borel sets, meaning that there is no infinite strictly descending sequence of Borel $\omega$-languages $X_{0}>_{W} X_{1}>_{W} X_{2}>_{W} \ldots$ These results ensure that, up to complementation and Wadge equivalence, the Borel Wadge hierarchy is a well ordering. Therefore, there exist a unique ordinal, called the height of the Borel Wadge hierarchy, and a mapping $d_{W}$ from the Borel Wadge hierarchy onto its height, called the Wadge degree, such that $d_{W}(X)<d_{W}(Y)$ if and only if $X<_{W} Y$, and $d_{W}(X)=d_{W}(Y)$ if and only if either $X \equiv_{W} Y$ or $X \equiv_{W} Y^{c}$, for every Borel $\omega$-languages $X$ and $Y$. The Borel Wadge hierarchy actually consists of an alternating succession of non-self-dual and self-dual sets with non-self-dual pairs at each limit level (as soon as finite alphabets are considered) [6,21].

Finally, the Borel Wadge hierarchy drastically refines the Borel hierarchy, since Borel sets of finite Borel ranks admit Wadge degrees ranging from 1 to the first fixpoint of the exponentiation of base $\omega_{1}$.

\subsection{The Wagner Hierarchy}

In 1979, Wagner described a classification of $\omega$-rational sets in terms of automata: the Wagner hierarchy $[7,13,22]$. This hierarchy has a height of $\omega^{\omega}$, and 
it is decidable. The Wagner degree of an $\omega$-rational language can indeed be computed by analyzing the graph of a Muller automaton accepting this language. Moreover, the Wagner hierarchy corresponds precisely to the restriction of the Wadge hierarchy to $\omega$-rational languages.

Selivanov gave a complete set theoretical description of the Wagner hierarchy in terms of boolean expressions [18], and Carton and Perrin [3,5] and Duparc and Riss [7] studied the algebraic properties of this hierarchy.

\section{The Wagner Degree as a syntactic invariant}

The syntactic pointed $\omega$-semigroup of an $\omega$-rational language is the unique (up to isomorphism) minimal (for the division) pointed $\omega$-semigroup recognizing this language. In this section, we show that the Wagner degree is a syntactic invariant: if two $\omega$-rational languages have the same syntactic image, then they also have the same Wagner degree. Therefore, the Wagner degree of every $\omega$-rational language can be characterized by some algebraic invariants on its syntactic image. The description of these invariants will be presented in the second paper.

We first recall the notion of syntactic $\omega$-semigroup. Given a subset $X$ of an $\omega$ semigroup $S$, the syntactic congruence of $X$, denoted by $\sim_{X}$, is the upper bound of the family of congruences recognizing $X$, if this upper bound still recognizes $X$, and is undefined otherwise. Whenever defined, the quotient $S(X)=S / \sim_{X}$ is called the syntactic $\omega$-semigroup of $X$, the quotient morphism $\mu: S \longrightarrow S(X)$ is the syntactic morphism of $X$, the set $\mu(X)$ is the syntactic image of $X$, and one has the property $\mu^{-1}(\mu(X))=X$. The pointed $\omega$-semigroup $(S(X), \mu(X))$ will be denoted by $\operatorname{Synt}(X)$. One can prove that the syntactic $\omega$-semigroup of an $\omega$-rational language is always defined [13], and that it satisfies the following minimality property:

Proposition 2.1 (see [13], Cor. 8.10, p. 117). Let L be an w-rational language. An $\omega$-semigroup $S$ recognizes $L$ if and only if $S(L)$ is a quotient of $S$.

Example 2.2. Let $K=\left(A^{*} a\right)^{\omega}$ be an $\omega$-language over the alphabet $A=\{a, b\}$. The morphism $\varphi: A^{\infty} \longrightarrow S$ given in Example 1.9 is the syntactic morphism of $K$. The $\omega$-subset $X=\left\{0^{\omega}\right\}$ of $S$ is the syntactic image of $K$.

Example 2.3. Let $B=\{a, b, c\}$ and let $L=\left(a\{b, c\}^{*} \cup\{b\}\right)^{\omega}$ be an $\omega$-language over $B$. The finite $\omega$-semigroup $T$ given in Example 1.6 is the syntactic $\omega$ semigroup of $L$. The morphism $\psi$ from $B^{\infty}$ into $T$ defined by $\psi(a)=a, \psi(b)=b$, and $\psi(c)=c$ is the syntactic morphism of $L$. The $\omega$-subset $Y=\left\{a^{\omega}\right\}$ of $T$ is the syntactic image of $L$.

We come to the main result of this section.

Proposition 2.4. Let $K$ and $L$ be two $\omega$-rational languages of $A^{\omega}$ and $B^{\omega}$, respectively. If $\operatorname{Synt}(K)$ divides $\operatorname{Synt}(L)$, then $K \leq_{W} L$. 
Proof. Let $\mu$ and $\nu$ be the syntactic morphisms of $K$ and $L$, respectively. If $\operatorname{Synt}(K)$ divides $\operatorname{Synt}(L)$, then there exist a pointed $\omega$-semigroup $(S, P)$, an injective morphism $\iota:(S, P) \longrightarrow \operatorname{Synt}(L)$, and a surjective morphism $\sigma:(S, P) \longrightarrow$ Synt $(K)$, as illustrated below:

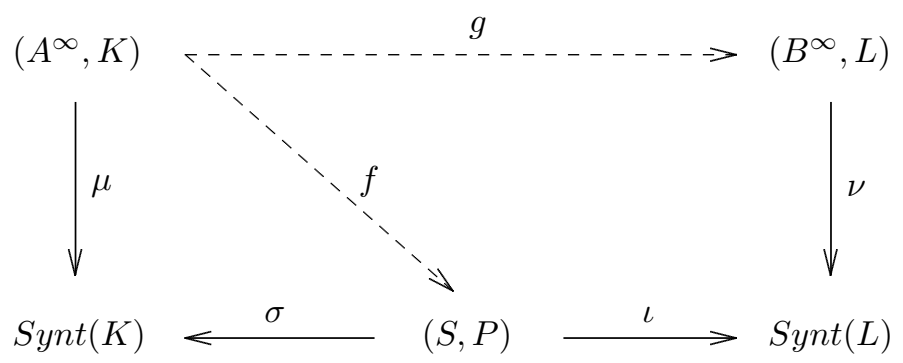

In particular, since $\sigma$ and $\iota$ are morphisms of pointed $\omega$-semigroups, the equalities $\sigma^{-1}(\mu(K))=P=\iota^{-1}(\nu(L))$ hold. Now, since $A^{\infty}$ is free and $\sigma$ is surjective, Corollary 4.7 of [13], p. 96 ensures that there exists a morphism of $\omega$-semigroups $f: A^{\infty} \rightarrow S$ such that $\sigma \circ f=\mu$. Moreover, since $\mu$ is the syntactic morphism of $K$, one has

$$
f^{-1}(P)=f^{-1}\left(\sigma^{-1}(\mu(K))\right)=\mu^{-1}(\mu(K))=K .
$$

Thus $f:\left(A^{\infty}, K\right) \longrightarrow(S, P)$ is a morphism of pointed $\omega$-semigroups. By composition, the mapping $\iota \circ f$ from $\left(A^{\infty}, K\right)$ into $\operatorname{Synt}(L)$ is a also morphism of pointed $\omega$-semigroups. Once again, since $A^{\infty}$ is free and $\nu$ is surjective, there exists a morphism of free $\omega$-semigroups $g=\left(g_{+}, g_{\omega}\right): A^{\infty} \longrightarrow B^{\infty}$ such that $\nu \circ g=\iota \circ f$. Moreover, since $\nu$ is the syntactic morphism of $L$, then

$$
g^{-1}(L)=g^{-1}\left(\nu^{-1}(\nu(L))\right)=f^{-1}\left(\iota^{-1}(\nu(L))\right)=f^{-1}(P)=K .
$$

Finally, it remains to prove that $g_{\omega}: A^{\omega} \longrightarrow B^{\omega}$ is continuous. Let $V B^{\omega}$ be an open set of $B^{\omega}$, with $V \subseteq B^{*}$. Since $g$ is a morphism, then $g_{\omega}^{-1}\left(V B^{\omega}\right)=g_{+}^{-1}(V) A^{\omega}$ which is an open set of $A^{\omega}$. Therefore $K \leq_{W} L$.

Corollary 2.5. If two $\omega$-rational languages have the same syntactic pointed $\omega$ semigroup, then they have the same Wagner degree.

Proof. An immediate consequence of Proposition 2.4.

\section{ThE $\mathbb{S} G$-HIERARCHY}

We define a reduction relation on pointed $\omega$-semigroups by means of an infinite two-player game. This reduction induces a hierarchy of Borel $\omega$-subsets, called the $\mathbb{S} G$-hierarchy. Many results of the Wadge theory [20] also apply in this framework and provide a detailed description of the $\mathbb{S} G$-hierarchy.

Let $S=\left(S_{+}, S_{\omega}\right)$ and $T=\left(T_{+}, T_{\omega}\right)$ be two $\omega$-semigroups, and let $X \subseteq S_{\omega}$ and $Y \subseteq T_{\omega}$ be two $\omega$-subsets. The game $\mathbb{S} \mathbb{G}((S, X),(T, Y))[2]$ is an infinite two-player 
game with perfect information, where Player I is in charge of $X$, Player II is in charge of $Y$, and players I and II alternately play elements of $S_{+}$and $T_{+} \cup\{-\}$, respectively. Player I begins. Unlike Player I, Player II is allowed to skip her turn - denoted by the symbol "_" -, provided she plays infinitely many moves. After $\omega$ turns each, players I and II produced respectively two infinite sequences $\left(s_{0}, s_{1}, \ldots\right) \in S_{+}^{\omega}$ and $\left(t_{0}, t_{1}, \ldots\right) \in T_{+}^{\omega}$. Player II wins $\mathbb{S} \mathbb{G}((S, X),(T, Y))$ if and only if $\pi_{S}\left(s_{0}, s_{1}, \ldots\right) \in X \Leftrightarrow \pi_{T}\left(t_{0}, t_{1}, \ldots\right) \in Y$. From this point onward, the game $\mathbb{S G}((S, X),(T, Y))$ will be denoted by $\mathbb{S} \mathbb{G}(X, Y)$ and the $\omega$-semigroups involved will always be known from the context. A play in this game is illustrated below.

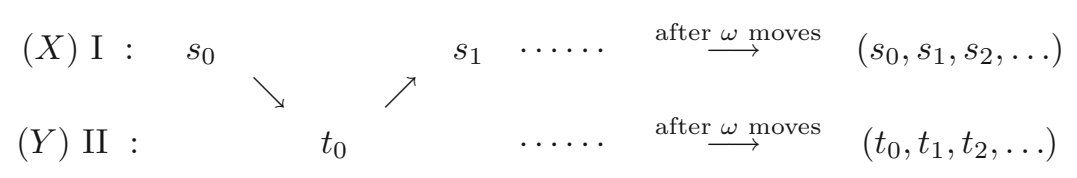

A player is said to be in position $s$ if the product of his/her previous moves $\left(s_{1}, \ldots, s_{n}\right)$ equals $s$. Strategies and winning strategies are defined as usual.

Now given two pointed $\omega$-semigroups $(S, X)$ and $(T, Y)$, we say that $X$ is $\mathbb{S} G$ reducible to $Y$, denoted by $X \leq_{S G} Y$, if and only if Player II has a winning strategy in $\mathbb{S} G(X, Y)$. We then naturally set $X \equiv_{S G} Y$ if and only if both $X \leq_{S G} Y$ and $Y \leq_{S G} X$, and also $X<_{S G} Y$ if and only if $X \leq_{S G} Y$ and $X \not \equiv_{S G} Y$. The relation $\leq_{S G}$ is reflexive and transitive, and $\equiv_{S G}$ is an equivalence relation. From this point forward, we say that $X$ and $Y$ are equivalent if $X \equiv_{S G} Y$. They are incomparable if $X \not_{S G} Y$ and $Y \not_{S G} X$.

First of all, we mention an elementary result showing that the empty set and the full space are incomparable and reducible to any other set. Some other basic properties follow.

Proposition 3.1. Let $S=\left(S_{+}, S_{\omega}\right)$ be an $\omega$-semigroup and let $X \subseteq S_{\omega}$.

(1) If $X \neq S_{\omega}$, then $\emptyset \leq_{S G} X$.

(2) If $X \neq \emptyset$, then $S_{\omega} \leq_{S G} X$.

(3) $\emptyset$ and $S_{\omega}$ are incomparable.

Proof.

(1) We describe a winning strategy for Player II in the game $\mathbb{S} G(\emptyset, X)$. At the end of the play, the infinite product of the infinite sequence played by I cannot belong to $\emptyset$. Hence, the winning strategy for II consists in playing an infinite sequence $\left(s_{0}, s_{1}, s_{2}, \ldots\right)$ such that $\pi_{S}\left(s_{0}, s_{1}, s_{2}, \ldots\right) \notin X$. This is indeed possible, since $X \neq S_{\omega}$.

(2) Similarly, we describe a winning strategy for Player II in the game $\mathbb{S G}$ $\left(S_{\omega}, X\right)$. At the end of the play, the infinite product of the infinite sequence played by I certainly belongs to $S_{\omega}$. Therefore, II wins the game by playing an infinite sequence $\left(s_{0}, s_{1}, s_{2}, \ldots\right)$ such that $\pi_{S}\left(s_{0}, s_{1}, s_{2}, \ldots\right) \in X$. This is possible, since $X \neq \emptyset$.

(3) We first show that Player II has no winning strategy in the game $\mathbb{S} G\left(\emptyset, S_{\omega}\right)$. At the end of the play, the infinite product of I's infinite sequence does 
clearly not belong to $\emptyset$, whereas the infinite product of II's infinite sequence obviously belongs to $S_{\omega}$. Therefore $\emptyset \not_{S G} S_{\omega}$. The same argument shows that $S_{\omega} \mathbb{Z}_{S G} \emptyset$.

Proposition 3.2. Let $(S, X)$ and $(T, Y)$ be two pointed $\omega$-semigroups.

(1) $X \leq_{S G} Y$ if and only if $X^{c} \leq_{S G} Y^{c}$.

(2) $X$ and $X^{c}$ are either equivalent or incomparable.

(3) If $X<_{S G} Y$, then $Y \mathbb{L}_{S G} X$ and $Y^{c} \mathbb{L}_{S G} X$.

Proof.

(1) By definition of the winning conditions of the $\mathbb{S} G$-game, a strategy is winning for Player II in $\mathbb{S} \mathbb{G}(X, Y)$ if and only if it is also winning for this same player in $\mathbb{S} \mathbb{G}\left(X^{c}, Y^{c}\right)$.

(2) Either $X \leq_{S G} X^{c}$, or $X \leq_{S G} X^{c}$. If $X \leq_{S G} X^{c}$, then (1) shows that $X^{c} \leq_{S G} X$, thus $X \equiv_{S G} X^{c}$. If $X \not_{S G} X^{c}$, then (1) shows that $X^{c} \not_{S G} X$, hence $X$ and $X^{c}$ are incomparable. Proposition 3.1 provides an example of two complementary incomparable sets, namely the empty and the full sets.

(3) If $X<_{S G} Y$, then $Y \mathbb{Z}_{S G} X$ by definition. Now, assume that $Y^{c} \leq_{S G}$ $X$. Then the relations $Y^{c} \leq_{S G} X$ and $X<_{S G} Y$ imply $Y^{c}<_{S G} Y$, a contradiction with (2).

Example 3.3. The $\omega$-subsets $X$ and $Y$ respectively given in Examples 2.2 and 2.3 satisfy $X \leq_{S G} Y$. Indeed, Player II has the following winning strategy in the game $\mathbb{S G}(X, Y)$. First of all, regardless of Player I's initial move, Player II answers with the element $a$. Afterwards, as long as Player I stays in position 1, Player II plays the element $c$. If this situation persists until the end of the play, players I and II respectively produce the elements $1^{\omega} \notin X$ and $a c^{\omega}=0 \notin Y$, and Player II wins the game. Now, if Player I reaches position 0, then Player II stays in position $a$, but answers as follows: when Player I plays 1, Player II plays $c$, and when Player I plays 0, Player II plays $c a$. Hence, at the end of the play, two cases may occur: either players I and II respectively produce the elements $01^{\omega}=1^{\omega} \notin X$ and $a c^{\omega}=0 \notin Y$, and therefore Player II wins the game; or players I and II respectively produce the elements $00^{\omega}=0^{\omega} \in X$ and $a(c a)^{\omega}=a^{\omega} \in Y$, and Player II also wins the game.

Borel Wadge determinacy implies the determinacy of $\mathbb{S} G$-games for any Borel winning $\omega$-subsets.

Theorem 3.4 (SGG-Borel Determinacy). Let $(S, X)$ and $(T, Y)$ be two Borel pointed $\omega$-semigroups. Then the game $\mathbb{S} G(X, Y)$ is determined.

Proof. By definition, since $(S, X)$ and $(T, Y)$ are Borel, the sets $\pi_{S}^{-1}(X)$ and $\pi_{T}^{-1}(Y)$ are Borel subsets of $S_{+}^{\omega}$ and $T_{+}^{\omega}$, respectively. In addition, a given player has a winning strategy in the game $\mathbb{S} G(X, Y)$ if and only if this same player has a winning strategy in the game $\mathbb{W}\left(\pi_{S}^{-1}(X), \pi_{T}^{-1}(Y)\right)$. Borel determinacy for Wadge games leads to the conclusion. 
We now extend the properties of the Wadge reduction to the $\mathbb{S} G$-reduction: the $\leq_{S G}$-antichains have length at most 2 , and the $\leq_{S G}$-relation is well-founded on Borel $\omega$-subsets. A description of the resulting hierarchy of Borel $\omega$-subsets follows.

Proposition 3.5. Let $(S, X)$ and $(T, Y)$ be two Borel pointed $\omega$-semigroups.

(1) (Wadge's Lemma) Either $X \leq_{S G} Y$ or $Y \leq_{S G} X^{c}$.

(2) If $X$ and $Y$ are incomparable, then $X \equiv_{S G} Y^{c}$.

(3) The $\leq_{S G \text {-antichains have length at most } 2 .}$

Proof.

(1) Either $X \leq_{S G} Y$ or $X \not_{S G} Y$. If $X \not_{S G} Y$, then Player II has no winning strategy in $\mathbb{S} G(X, Y)$. Hence, by determinacy, Player I has a winning strategy $\sigma$ in this game. This induces the following winning strategy for Player II in $\mathbb{S G}\left(Y, X^{c}\right)$ : she plays $\sigma(\varepsilon)$ on her first move, and then, she answers to every current position $\left(x_{0}, \ldots, x_{n}\right)$ of Player I by the move $\sigma\left(x_{0} \ldots x_{n-1}\right)$. Thus $Y \leq_{S G} X^{c}$.

(2) If $X \not_{S G} Y$ and $Y \mathbb{Z}_{S G} X$, then (1) implies both $Y \leq_{S G} X^{c}$ and $X \leq_{S G}$ $Y^{c}$. Therefore $Y \leq_{S G} X^{c}$ and $X^{c} \leq_{S G} Y$, which means $X^{c} \equiv_{S G} Y$.

(3) Let $X, Y$, and $Z$ be $\omega$-subsets such that $X \not_{S G} Y$ and $Y \mathbb{Z}_{S G} Z$. Then point (1) shows that $Y \leq X^{c}$ and $Z \leq_{S G} Y^{c}$. Hence $Z \leq_{S G} Y^{c}$ and $Y^{c} \leq X$, and therefore $Z \leq_{S G} X$.

Proposition 3.6 (Martin, Monk). The partial ordering $\leq_{S G}$ is well-founded on Borel pointed $\omega$-semigroups.

Proof. Towards a contradiction, assume there exists an infinite strictly descending sequence of Borel pointed $\omega$-semigroups $\left(\left(S_{i}, X_{i}\right)\right)_{i>0}$, i.e. $X_{0}>_{S G} X_{1}>_{S G}$ $X_{2}>_{S G} \ldots$, where $S_{i}=\left(S_{i,+}, S_{i, \omega}\right)$ and $X_{i} \subseteq S_{i, \omega}$ for all $i \geq 0$. By Proposition 3.2 (3), the relation $X_{n}>_{S G} X_{n+1}$ implies $X_{n} \not_{S G} X_{n+1}$ and $X_{n}^{c} \not_{S G} X_{n+1}$, for all $n \geq 0$. Therefore, by determinacy, Player I has the winning strategies $\sigma_{n}^{0}$ and $\sigma_{n}^{1}$ in the respective games $\mathbb{S} G\left(X_{n}, X_{n+1}\right)$ and $\mathbb{S} \mathbb{G}\left(X_{n}^{c}, X_{n+1}\right)$, for all $n \geq 0$. Now, for any $\alpha \in\{0,1\}^{\omega}$, consider the infinite sequence of strategies $\left(\sigma_{n}^{\alpha(n)}\right)_{n \geq 0}$, and the infinite sequence of games $\left(\mathbb{S G}\left(X_{n}^{c(\alpha(n))}, X_{n+1}\right)\right)_{n \geq 0}$ related as follows: in the game $\mathbb{S} G\left(X_{k}^{c(\alpha(k))}, X_{k+1}\right)$, Player I applies his winning strategy $\sigma_{k}^{\alpha(k)}$ and Player II copies Player I's moves of the next game $\mathbb{S} G\left(X_{k+1}^{c(\alpha(k+1))}, X_{k+2}\right)$. Therefore, in the first game, Player I applies his winning strategy $\sigma_{0}^{\alpha(0)}$. Since it is a strategy for Player I, it gives the first letter $a_{0}^{0}$ before Player II has ever played anything. Then Player II copies Player I's first move $a_{0}^{1}$ of the second game, and Player I answers with his winning strategy. And so on and so forth for every move and every game. This infinite sequence of games is illustrated below. Big and small 
arrows respectively denote the actions of playing and copying.

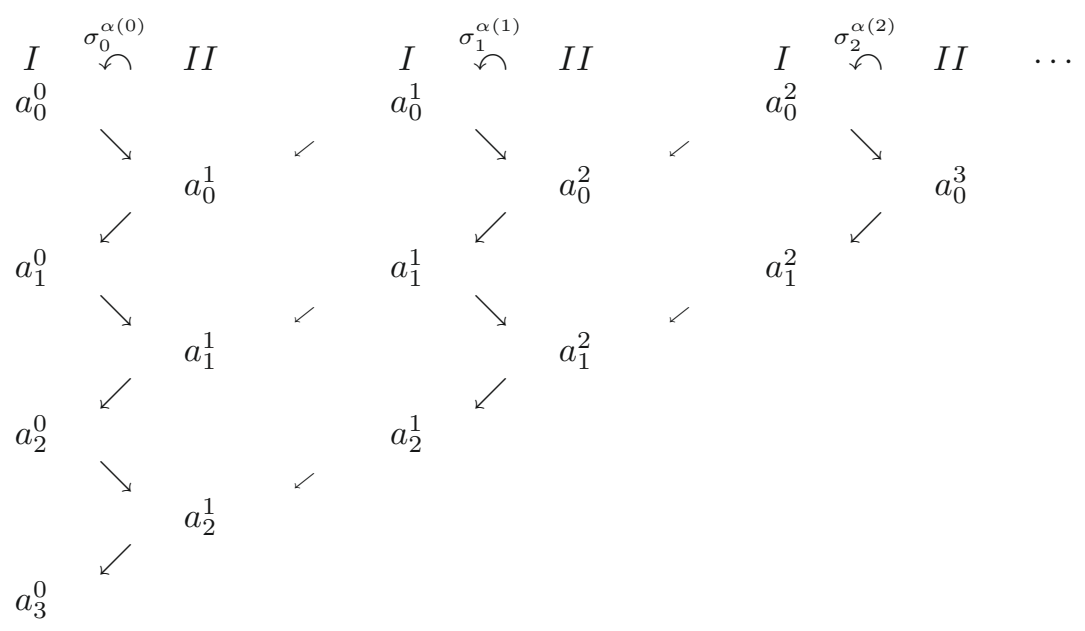

Let $x_{\alpha}=a_{0}^{0} a_{1}^{0} a_{2}^{0} \ldots$ be the infinite word played by Player I in the first game, let

$$
\varphi:\{0,1\}^{\omega} \longrightarrow S_{0,+}^{\omega}
$$

be defined by $\varphi(\alpha)=x_{\alpha}$, and let

$$
\psi=\pi_{S_{0}} \circ \varphi:\{0,1\}^{\omega} \longrightarrow S_{0, \omega}
$$

be defined by $\psi(\alpha)=\pi_{S_{0}}\left(x_{\alpha}\right)=\pi_{S_{0}}\left(a_{0}^{0}, a_{1}^{0}, a_{2}^{0}, \ldots\right)$. We show that $\varphi$ is continuous. By definition of these chained games, the $k$ first letters of $x_{\alpha}$ only depend on the $k$ first letters of $\alpha$, since we completely do not need the games number $k+1, k+2, \ldots$ to determine $x_{\alpha}[0, k]$. Thus, for any $U \subseteq S_{0,+}^{*}$, one has $\varphi^{-1}\left(U S_{0,+}^{\omega}\right)=V\{0,1\}^{\omega}$, with $V \subseteq\{0,1\}^{*}$, hence the preimage by $\varphi$ of an open set is an open set, which proves that $\varphi$ is continuous. Now consider $F=\psi^{-1}\left(X_{0}\right)=\varphi^{-1}\left(\pi_{S_{0}}^{-1}\left(X_{0}\right)\right)$. By construction of these chained games, $F$ is a flip set, because if $\alpha$ and $\alpha^{\prime}$ only differ by one position (meaning if there exists a unique $i$ such that $\alpha(i) \neq \alpha^{\prime}(i)$ ), then $\alpha \in F$ if and only if $\alpha^{\prime} \notin F$. On the other hand, the set $F$ is also Borel, since $\pi_{S_{0}}^{-1}\left(X_{0}\right)$ is by definition Borel and $\varphi$ is continuous.

The collection of Borel $\omega$-subsets ordered by the $\leq_{S G}$-relation is called the $\mathbb{S G}$-hierarchy, in order to underline the semigroup approach. Notice that the restriction of the $\mathbb{S} G$-hierarchy to Borel $\omega$-subsets of free $\omega$-semigroups is exactly the Borel Wadge hierarchy. When restricted to the Borel $\omega$-subsets of finite $\omega$ semigroups, this hierarchy will be called the $\mathbb{F S G}$-hierarchy, in order to underline the finiteness of the $\omega$-semigroups involved. Propositions 3.5 and 3.6 show that, up to complementation and $\leq_{S G}$-equivalence, the $\mathbb{S} G$-hierarchy is a well ordering. Therefore, there exist a unique ordinal, called the height of the $\mathbb{S} G$-hierarchy, and a mapping $d_{S G}$ from the $\mathbb{S} G$-hierarchy onto its height, called the $\mathbb{S} \mathbb{G}$-degree, 


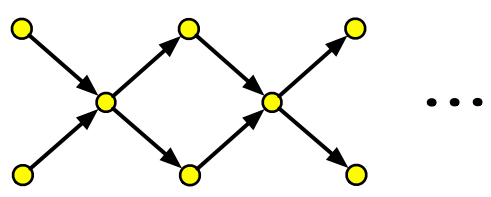

Figure 1. The shape of the $\mathbb{S} G$-hierarchy.

such that $d_{S G}(X)<d_{S G}(Y)$ if and only if $X<_{S G} Y$, and $d_{S G}(X)=d_{S G}(Y)$ if and only if either $X \equiv_{S G} Y$ or $X \equiv_{S G} Y^{c}$, for every Borel $\omega$-subsets $X$ and $Y$. It directly follows from the Wadge analysis that the $\mathbb{S} G$-hierarchy has the same familiar "scaling shape" as the Borel or Wadge hierarchies: an increasing sequence of non-self-dual sets with self-dual sets in between, and with non-self-dual pairs at each limit level (as soon as finite alphabets are considered), as illustrated in

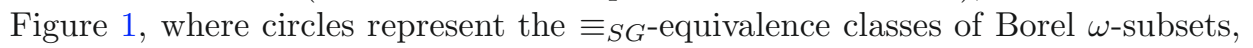
and arrows stand for the $<_{S G}$-relation.

\section{The $\mathbb{F} \mathbb{S} G$ AND the Wagner hiERARChIES}

This section shows that the $\mathbb{F} \mathbb{S} G$-hierarchy is precisely the algebraic counterpart of the Wagner hierarchy. Consequently, the $\mathbb{F S G}$-hierarchy has a height of $\omega^{\omega}$, and it is decidable.

Let $S=\left(S_{+}, S_{\omega}\right)$ be a finite $\omega$-semigroup, and let $\varphi: A^{\infty} \longrightarrow S$ be a surjective morphism of $\omega$-semigroups, for some finite alphabet $A$. Then every $\omega$-subset $X$ of $S_{\omega}$ can be lifted on an $\omega$-rational language $\varphi^{-1}(X)$ of $A^{\omega}$. The next proposition proves that this lifting induces an embedding from the $\mathbb{F S G}$-hierarchy into the Wagner hierarchy.

Proposition 4.1. Let $(S, X)$ and $(T, Y)$ be two finite pointed $\omega$-semigroups., and let $\varphi: A^{\infty} \longrightarrow S$ and $\psi: B^{\infty} \longrightarrow T$ be two surjective morphisms of $\omega$-semigroups, where $A$ and $B$ are finite alphabets. Then

$$
X \leq_{S G} Y \text { if and only if } \varphi^{-1}(X) \leq_{W} \psi^{-1}(Y) .
$$

Proof.

$(\Rightarrow)$ Given a winning strategy $\sigma$ for Player II in $\mathbb{S} \mathbb{G}(X, Y)$, we describe a winning strategy $\tau$ for this same player in the game $\mathbb{W}\left(\varphi^{-1}(X), \psi^{-1}(Y)\right)$. Assume Player I is in position $\left(a_{0}, \ldots, a_{n}\right)$. Then II computes the move $\sigma\left(\varphi\left(a_{0}\right), \ldots, \varphi\left(a_{n}\right)\right)$. If it is not a skipping move, she chooses a finite word $v_{n}$ such that $\psi\left(v_{n}\right)=$ $\sigma\left(\varphi\left(a_{0}\right), \ldots, \varphi\left(a_{n}\right)\right)$, keeps it in mind while she finishes to play letter by letter the finite words she had previously chosen, and then plays $v_{n}$ letter by letter. If it is a skipping move, then either she finishes to play letter by letter the finite words she 
had previously chosen, or she skips her turn if it is already done. This strategy is illustrated below:

SG-game

\begin{tabular}{ccc}
$I(X)$ & $\stackrel{\sigma}{\curvearrowright}$ & $I I(Y)$ \\
\hline$\varphi\left(a_{0}\right)$ & & $\sigma\left(\varphi\left(a_{0}\right)\right)$ \\
$\varphi\left(a_{1}\right)$ & & $\sigma\left(\varphi\left(a_{0}\right), \varphi\left(a_{1}\right)\right)$
\end{tabular}

Wadge game

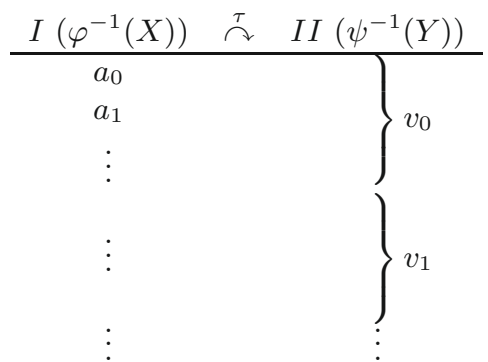

It remains to prove that this strategy is winning for Player II. Since $\varphi$ and $\psi$ are surjective morphisms of $\omega$-semigroups, one obtains

$$
\begin{aligned}
a_{0} a_{1} a_{2} \cdots \in \varphi^{-1}(X) & \Leftrightarrow \varphi\left(a_{0} a_{1} a_{2} \cdots\right) \in X \\
& \Leftrightarrow \varphi\left(a_{0}\right) \varphi\left(a_{1}\right) \varphi\left(a_{2}\right) \cdots \in X \\
& \Leftrightarrow \sigma\left(\varphi\left(a_{0}\right)\right) \sigma\left(\varphi\left(a_{0}\right), \varphi\left(a_{1}\right)\right) \cdots \in Y \\
& \Leftrightarrow \psi\left(v_{0}\right) \psi\left(v_{1}\right) \psi\left(v_{2}\right) \cdots \in Y \\
& \Leftrightarrow v_{0} v_{1} v_{2} \cdots \in \psi^{-1}(Y) .
\end{aligned}
$$

Consequently, $\varphi^{-1}(X) \leq_{W} \psi^{-1}(Y)$.

$(\Leftarrow)$ Given a winning strategy $\sigma$ for Player II in $\mathbb{W}\left(\varphi^{-1}(X), \psi^{-1}(Y)\right)$, we describe a winning strategy $\tau$ for this same player in $\mathbb{S G}(X, Y)$. Assume Player I is in position $\left(s_{0}, \ldots, s_{n}\right)$. Then Player II chooses a finite word $u_{n}=u_{n, 0} \ldots u_{n, k_{n}}$ of $\varphi^{-1}\left(s_{n}\right)$, and computes the successive elements of $B^{\omega} \sigma\left(u_{0}, \ldots, u_{n-1}, u_{n, 0}\right)$, $\sigma\left(u_{0}, \ldots, u_{n-1}, u_{n, 0}, u_{n, 1}\right)$, and so on and so forth. After that, she successively plays the images by $\psi$ of these moves when they are non-skipping, and she skips her turn otherwise. This strategy is illustrated as follows:

Wadge game

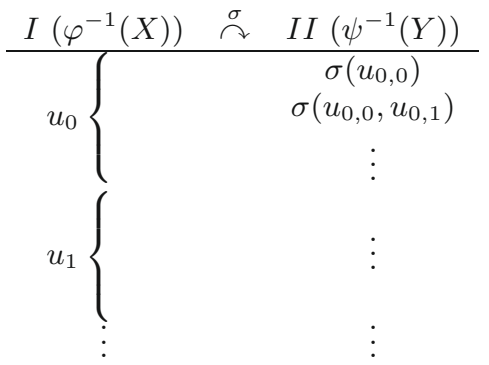

$\mathbb{S} G$-game

\begin{tabular}{ccc}
$I(X)$ & $\stackrel{\tau}{\curvearrowright}$ & $I I(Y)$ \\
\hline$s_{0}$ & $\psi \circ \sigma\left(u_{0,0}\right)$ \\
$s_{1}$ & $\psi \circ \sigma\left(u_{0,0}, u_{0,1}\right)$
\end{tabular}


It remains to prove that the strategy $\tau$ is winning for Player II. Since $\varphi$ and $\psi$ are surjective morphisms of $\omega$-semigroups, one has

$$
\begin{aligned}
s_{0} s_{1} s_{2} \cdots \in X & \Leftrightarrow \varphi\left(u_{0}\right) \varphi\left(u_{1}\right) \varphi\left(u_{2}\right) \ldots \in X \\
& \Leftrightarrow u_{0} u_{1} u_{2} \ldots \in \varphi^{-1}(X) \\
& \Leftrightarrow u_{0,0} \cdots u_{0, k_{0}} u_{1,0} \ldots u_{1, k_{1}} \ldots \in \varphi^{-1}(X) \\
& \Leftrightarrow \sigma\left(u_{0,0}\right) \sigma\left(u_{0,0}, u_{0,1}\right) \ldots \in \psi^{-1}(Y) \\
& \Leftrightarrow \psi\left(\sigma\left(u_{0,0}\right) \sigma\left(u_{0,0}, u_{0,1}\right) \ldots\right) \in Y \\
& \Leftrightarrow \psi\left(\sigma\left(u_{0,0}\right)\right) \psi\left(\sigma\left(u_{0,0}, u_{0,1}\right)\right) \ldots \in Y .
\end{aligned}
$$

Therefore, $X \leq_{S G} Y$.

The previous proposition shows that the Wadge reduction on $\omega$-rational languages and the $\mathbb{S} \mathbb{G}$-reduction on $\omega$-subsets recognizing these languages coincide. This property holds in particular for $\omega$-rational languages and their syntactic images, as mentioned in Corollary 4.2 below. In addition, Corollary 4.2 and Proposition 2.4 prove that the $\mathbb{S} G$-relation on subsets of $\omega$-semigroups is weaker than the division relation, and is the appropriate algebraic counterpart of the Wadge reduction on $\omega$-rational languages.

Corollary 4.2. Let $K$ and $L$ be two $\omega$-rational languages and let $\mu(K)$ and $\nu(L)$ be their syntactic images. Then $K \leq_{W} L$ if and only if $\mu(K) \leq_{S G} \nu(L)$.

Proof. Since $\mu$ and $\nu$ are syntactic morphisms, one has $\mu^{-1}(\mu(K))=K$ and $\nu^{-1}(\nu(L))=L$. Proposition 4.1 leads to the conclusion.

Example 4.3. Consider the $\omega$-subsets $X$ and $Y$, and the $\omega$-rational languages $K$ and $L$ respectively given in Examples 2.2 and 2.3. Example 3.3 shows that $X \leq_{S G} Y$, and hence $K \leq_{W} L$.

As another consequence, the $\mathbb{S} G$-degree of an $\omega$-subset is invariant under surjective morphism, and in particular under syntactic morphism. Therefore, syntactic finite pointed $\omega$-semigroups are minimal representatives of their $\leq_{S G}$-equivalence class.

Corollary 4.4. Let $\mu: S \longrightarrow T$ be a surjective morphism of finite $\omega$-semigroups, let $Y \subseteq T_{\omega}$, and let $X=\mu^{-1}(Y)$. Then $X \equiv_{S G} Y$.

Proof. Let $\varphi: S_{+}^{\infty} \longrightarrow S$ be the canonical morphism of $\omega$-semigroups associated with $S$, and let $\psi=\mu \circ \varphi: S_{+}^{\infty} \longrightarrow T$. The mapping $\psi$ is a surjective morphism of $\omega$-semigroups. It satisfies $\psi^{-1}(Y)=\varphi^{-1} \circ \mu^{-1}(Y)=\varphi^{-1}(X)$, thus in particular, $\varphi^{-1}(X) \equiv_{W} \psi^{-1}(Y)$. Proposition 4.1 then shows that $X \equiv_{S G} Y$.

Finally, the following theorem proves that the Wagner and the $\mathbb{F S G}$-hierarchies are isomorphic. The required isomorphism is the mapping which associates every $\omega$-rational language with its syntactic image. Therefore, the Wagner degree of an $\omega$-rational language and the $\mathbb{S} \mathbb{G}$-degree of its syntactic image are the same. 
Theorem 4.5. The Wagner hierarchy and the $\mathbb{F} \mathbb{S G}$-hierarchy are isomorphic.

Proof. Consider the mapping from the Wagner hierarchy into the $\mathbb{S G}$-hierarchy which associates every $\omega$-rational language with its syntactic image. We prove that this mapping is an embedding. Let $K$ and $L$ be two $\omega$-rational languages, and let $X=\mu(K)$ and $Y=\nu(L)$ be their syntactic images. Corollary 4.2 ensures that $K \leq_{W} L$ if and only if $X \leq_{S G} Y$. We now show that, up to $\equiv_{S G}$-equivalence, this mapping is onto. Let $X$ be an $\omega$-subset of a finite $\omega$-semigroup $S=\left(S_{+}, S_{\omega}\right)$, let $\mu: S \longrightarrow S(X)$ be the syntactic morphism of $X$, and let $Y=\mu(X)$ be its syntactic image. Corollary 4.4 ensures that $X \equiv_{S G} Y$. Now, let also $\varphi: S_{+}^{\infty} \longrightarrow S$ be the canonical morphism associated with $S_{+}$, and let $L=\varphi^{-1}(X)$. Then the morphism of $\omega$-semigroups $\psi=\mu \circ \varphi: S_{+}^{\infty} \longrightarrow S(X)$ is the syntactic morphism of $L[13]$, and one has $\psi(L)=Y \equiv_{S G} X$.

As a corollary, we show that the $\mathbb{F} \mathbb{S} G$-hierarchy is decidable: for every $\omega$-subset $X$ of the hierarchy, one can effectively compute the Cantor normal form of base $\omega$ of the ordinal $d_{S G}(X)$.

Corollary 4.6. The $\mathbb{F S G - h i e r a r c h y ~ h a s ~ h e i g h t ~} \omega^{\omega}$, and it is decidable.

Proof. By the previous theorem, the $\mathbb{F S G}$-hierarchy and the Wagner hierarchy have the same height, namely $\omega^{\omega}$. In addition, given an $\omega$-subset $X$ of a finite $\omega$-semigroup $S=\left(S_{+}, S_{\omega}\right)$, one can effectively compute the $\mathbb{S} \mathbb{G}$-degree of $X$ as follows. Let $\varphi: S_{+}^{\infty} \longrightarrow S$ be the canonical morphism associated with $S_{+}$, and let $L=\varphi^{-1}(X)$. Theorem 4.5 shows that the $\mathbb{S} \mathbb{G}$-degree of $X$ is equal to the Wagner degree of $L$. Furthermore, the Wagner degree of $L$ can be effectively computed as follows. First, one can effectively compute an $\omega$-rational expression describing $L=$ $\varphi^{-1}(X)$ ([13], Cor. 7.4, p. 110). Next, one can shift from this rational expression to some finite Muller automaton recognizing $L$ (see [13], Chap. I, Sects. 10.1, 10.3, and 10.4). Finally, the Wagner degree of the $\omega$-language recognized by a finite Muller automaton is effectively computable [22].

Example 4.7. Consider the $\omega$-subsets $X$ and $Y$ respectively given in Examples 2.2 and 2.3. The algorithm given in the second paper will show that $d_{S G}(X)=\omega$ and $d_{S G}(Y)=\omega^{2}$. In addition, since these sets are the syntactic images of the $\omega$ languages $K=\left(A^{*} a\right)^{\omega}$ and $L=\left(a\{b, c\}^{*} \cup\{b\}\right)^{\omega}$, Theorem 4.5 shows that this result can also be obtained by computing the Wagner degrees of the $\omega$-languages $K$ and $L[7,13,22]$.

\section{Conclusion}

This work is a first step towards the description of the algebraic counterpart of the Wagner hierarchy. First, we proved that the Wagner degree of $\omega$-rational languages is a syntactic invariant. Afterwards, we defined a reduction relation on finite pointed $\omega$-semigroups, and showed that the resulting algebraic hierarchy is indeed isomorphic to the Wagner hierarchy. Consequently, this hierarchy has a height of $\omega^{\omega}$, and it is decidable. But the decidability procedure presented in 
Corollary 4.6 relies on Wagner's naming procedure, and in this sense withdraws from the purely algebraic context.

The second paper fills this gap by describing the algebraic invariants which characterize the Wagner degree of $\omega$-rational languages. More precisely, we introduce a graph representation of finite pointed $\omega$-semigroups, as well as a graphical decidability procedure computing the $\mathbb{S} \mathbb{G}$-degree of every such algebraic structure. Therefore, the Wagner degree of every $\omega$-rational language can be computed directly on its syntactic image.

Acknowledgements. The authors wish to express their deep gratitude to Jean-Eric Pin for his very significant contribution to this paper, in particular as the PhD supervisor of the first author.

\section{REFERENCES}

[1] A. Andretta, Equivalence between Wadge and Lipschitz determinacy. Ann. Pure Appl. Logic 123 (2003) 163-192.

[2] J. Cabessa and J. Duparc, An infinite game over $\omega$-semigroups. In Foundations of the Formal Sciences V, Infinite Games, edited by S. Bold, B. Löwe, T. Räsch, J. van Benthem, Studies in Logic 11, College Publications, London (2007) 63-78.

[3] O. Carton and D. Perrin, Chains and superchains for $\omega$-rational sets, automata and semigroups. Int. J. Algebra Comput. 7 (1997) 673-695.

[4] O. Carton and D. Perrin, The Wadge-Wagner hierarchy of $\omega$-rational sets. In Automata, languages and programming (Bologna, 1997). Lect. Notes Comput. Sci. 1256 (1997) 17-35.

[5] O. Carton and D. Perrin, The Wagner hierarchy. Int. J. Algebra Comput. 9 (1999) 597-620.

[6] J. Duparc, Wadge hierarchy and Veblen hierarchy. I. Borel sets of finite rank. J. Symbolic Logic 66 (2001) 56-86.

[7] J. Duparc and M. Riss, The missing link for $\omega$-rational sets, automata, and semigroups. Int. J. Algebra Comput. 16 (2006) 161-185.

[8] R.E. Ladner, Application of model theoretic games to discrete linear orders and finite automata. Inform. Control 33 (1977) 281-303.

[9] D.A. Martin, Borel determinacy. Ann. Math. 102 (1975) 363-371.

[10] R.t McNaughton and S.A. Papert, Counter-Free Automata (M.I.T. research monograph No. 65). The MIT Press (1971).

[11] D. Perrin and J.-E. Pin, First-order logic and star-free sets. J. Comput. System Sci. 32 (1986) 393-406.

[12] D. Perrin and J.-E. Pin, Semigroups and automata on infinite words. In Semigroups, formal languages and groups (York, 1993). Kluwer Acad. Publ., Dordrecht (1995) 49-72.

[13] D. Perrin and J.-E. Pin, Infinite words. Pure and Applied Mathematics 141, Elsevier (2004).

[14] J.-E. Pin, Logic, semigroups and automata on words. Ann. Math. Artif. Intell. 16 (1996) 343-384.

[15] J.-E. Pin, Positive varieties and infinite words. in edited by Latin'98, edited by C.L. Lucchesi and A.V. Moura. Lect. Notes Comput. Sci. 1380 (1998) 76-87.

[16] J. Sakarovitch, Monoïdes pointés. Semigroup Forum 18 (1979) 235-264.

[17] M.P. Schützenberger, On finite monoids having only trivial subgroups. Inform. Control 8 (1965) 190-194.

[18] V. Selivanov, Fine hierarchy of regular $\omega$-languages. Theoret. Comput. Sci. 191 (1998) $37-59$.

[19] W. Thomas, Star-free regular sets of $\omega$-sequences. Inform. Control 42 (1979) 148-156. 
[20] W.W. Wadge, Degrees of complexity of subsets of the baire space. Notice A.M.S. (1972) A714-A715

[21] W.W. Wadge, Reducibility and determinateness on the Baire space. Ph.D. thesis, University of California, Berkeley (1983).

[22] K. Wagner, On $\omega$-regular sets. Inform. Control 43 (1979) 123-177.

[23] T. Wilke, An Eilenberg theorem for $\infty$-languages. In Automata, languages and programming (Madrid, 1991). Lect. Notes Comput. Sci. 510 (1991) 588-599.

[24] T. Wilke and H. Yoo, Computing the Wadge degree, the Lifshitz degree, and the Rabin index of a regular language of infinite words in polynomial time, in TAPSOFT '95: Theory and Practive of Software Development, edited by P.D. Mosses, M. Nielsen and M.I. Schwartzbach. Lect. Notes Comput. Sci. 915 (1995) 288-302.

Communicated by Ch. Choffrut.

Received Avril 10, 2008. Accepted December 18, 2008. 